\title{
A Method for Order/Degree Problem Based on Graph Symmetry and Simulated Annealing with MPI/OpenMP Parallelization
}

\author{
Masahiro Nakao \\ RIKEN Center for Computational \\ Science \\ Hyogo, Japan
}

\author{
Hitoshi Murai \\ RIKEN Center for Computational \\ Science \\ Hyogo, Japan
}

\author{
Mitsuhisa Sato \\ RIKEN Center for Computational \\ Science \\ Hyogo, Japan
}

\begin{abstract}
The network topology in various systems, such as large-scale data centers, high-performance computing systems, and Network on Chip, is strongly related to network latency. Designing a network topology with low latency can be defined as an order/degree problem (ODP) in graph theory by modeling the network topology as an undirected graph. This study proposes a method for efficiently solving ODPs based on graph symmetry and simulated annealing (SA). This method makes the network topology symmetrical, thereby improving the solution search performance of SA and drastically reducing the calculation time. The proposed method is applied to several problems from an international competition for ODPs called Graph Golf to find network topologies with sufficiently low latency. The symmetry-based calculation achieves a speed up of 31.76 times for one of the problems. Furthermore, to reduce calculation time, the proposed method is extended to use hybrid parallelization with MPI and OpenMP. As a result, a maximum speed up of 209.80 times was achieved on 20 compute nodes consisting of $400 \mathrm{CPU}$ cores. Even faster performance was achieved by combining the symmetry-based calculation and hybrid parallelization.
\end{abstract}

\section{CCS CONCEPTS}

- Networks $\rightarrow$ Topology analysis and generation;

\section{KEYWORDS}

network topology, high-performance computing, optimization problem, simulated annealing

\section{ACM Reference Format:}

Masahiro Nakao, Hitoshi Murai, and Mitsuhisa Sato. 2019. A Method for Order/Degree Problem Based on Graph Symmetry and Simulated Annealing with MPI/OpenMP Parallelization. In International Conference on High Performance Computing in Asia-Pacific Region (HPC Asia 2019), fanuary 14 - 16, 2019, Guangzhou, China. ACM, New York, NY, USA, 10 pages. https://doi.org/10.1145/3293320.3293325

\section{BACKGROUND}

Network topology, such as those of network cables in large-scale data centers and high-performance computing systems and wiring

Permission to make digital or hard copies of part or all of this work for personal or classroom use is granted without fee provided that copies are not made or distributed for profit or commercial advantage and that copies bear this notice and the full citation on the first page. Copyrights for third-party components of this work must be honored. For all other uses, contact the owner/author(s).

HPC Asia 2019, January 14-16, 2019, Guangzhou, China

(C) 2019 Copyright held by the owner/author(s).

ACM ISBN 978-1-4503-6632-8/19/01.

https://doi.org/10.1145/3293320.3293325 in chips for Network on Chip (NoC), greatly affects the latency of a system $[1,7,10,18]$. One way to reduce latency is to adopt a network topology with a small diameter and the average shortest path length (ASPL)[12]. The network topology can be modeled as an undirected graph and defined as an order/degree problem (ODP) in graph theory. An ODP is the problem of finding an undirected graph with the smallest diameter and ASPL that satisfies the given number of vertices and degrees. Hereafter, an undirected graph is simply referred to as a "graph."

There is an international competition for ODPs organized by the National Institute of Informatics in Japan called Graph Golf[13]. Graph Golf, which has been held every year since 2015, gives several problems with various combinations of numbers of vertices and degrees. For problems in the General Graph category, vertices can be placed freely, and for those in the Grid Graph category, vertices must be placed on a grid. This study considers problems in the General Graph category. Participants of Graph Golf can upload their graphs on the official website within a limited period (May 14, 2018, to October 14, 2018, for this year's competition).

Although the definition of ODPs is very simple, there are no efficient methods for solving them. ODPs are difficult for the following reasons. (1) The number of graphs satisfying the given number of vertices and degrees is enormous. When an ODP is considered as a discrete optimization problem, it is difficult to find the best solution because the problem has many local optima. (2) The calculation time required for obtaining the diameter and ASPL is enormous because it is necessary to search for the shortest path from all vertices to all other vertices. This is the so-called all-pairs shortest-paths (APSP) problem. In order to solve an APSP problem, it is sufficient to perform a breadth-first search (BFS) for all vertices. The calculation complexity for an APSP problem with $n$ vertices and $d$ degrees is $O\left(n^{2} d\right)^{1}$. Therefore, as the number of vertices increases, the calculation time becomes very large.

The present study proposes a method for solving ODPs. It's main features are as follows. (1) By using simulated annealing (SA) [9, 11], a general optimization framework, the solution search does not easily fall into local optima. (2) By giving symmetry to a graph, SA can find solutions more efficiently, greatly reducing calculation time. (3) Hybrid parallelization with MPI and OpenMP is applied to further reduce calculation time on a cluster system.

The remainder of this paper is structured as follows. Section 2 gives an overview of ODPs and reviews related work. Section 3 describes the design of the proposed method for solving ODPs.

\footnotetext{
${ }^{1}$ The Floyd-Warshal algorithm[6, 20] and the Seidel algorithm[17] for APSP problems in a dense network have calculation complexities of $O\left(n^{3}\right)$ and $O\left(n^{2.376} \log n\right)$, respectively. However, since Graph Golf uses a relatively sparse network, a method based on BFS is faster.
} 

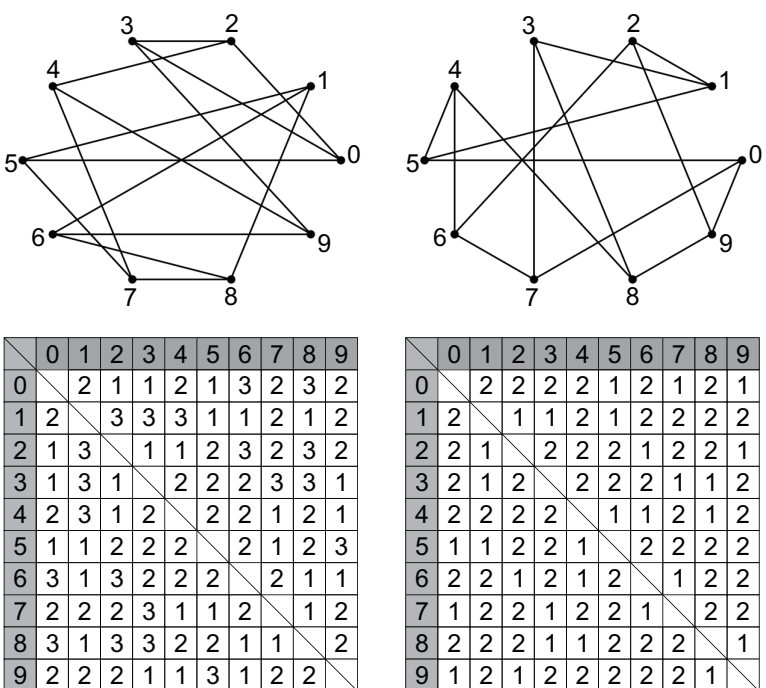

(a) Diameter $=3$, ASPL $=1.89$

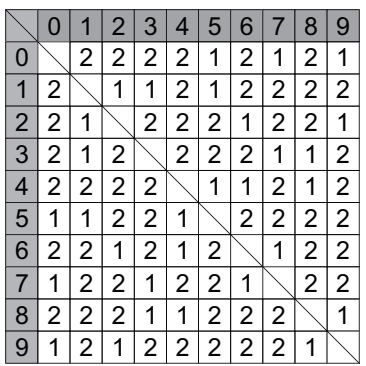

(b) Diameter=2, ASPL $=1.67$

Figure 1: Examples of graph $(n=10, d=3)$

Sections 4 and 5 evaluate the search solution performance and calculation time, respectively. Section 6 summarizes the results and suggests areas for future research.

\section{ORDER/DEGREE PROBLEMS}

\subsection{Overview}

An ODP is the problem of minimizing the diameter and ASPL of a graph that satisfies the given number of vertices and degrees. From the numbers of vertices $n$ and degrees $d$, the theoretical diameter lower bound $K_{n, d}$ and the ASPL lower bound $L_{n, d}$ are calculated as follows[3]:

$$
\begin{gathered}
K_{n, d}= \begin{cases}\left\lceil\frac{n-1}{2}\right\rceil & \text { if } d=2 \\
\left\lceil\log _{d-1}\left(\frac{(n-1)(d-2)}{d}\right)+1\right\rceil & \text { if } d>2\end{cases} \\
L_{n, d}= \begin{cases}\frac{S_{n, d}+K_{n, d} R_{n, d}}{n-1} & \text { if } K_{n, d}=1 \\
\text { if } K_{n, d} \geq 2\end{cases} \\
S_{n, d}=\sum_{i=1}^{K_{n, d}-1} i d(d-1)^{i-1} \\
R_{n, d}=n-1-\sum_{i=1}^{K_{n, d}-1} d(d-1)^{i-1}
\end{gathered}
$$

Fig. 1 shows examples of a graph with $n=10$ and $d=3$, and the corresponding distance matrices. Fig. 1a shows a graph made by connecting edges with vertices randomly, called a random graph, and Fig. 1b shows a graph for which both the diameter and ASPL are the respective lower bounds. The distance matrices, which are

symmetrical, show the distance from each vertex to all other vertices. The diameter is the maximum value of the elements in a distance matrix. The ASPL is obtained by dividing the sum of the values in a distance matrix by the number of elements without diagonal elements $\left(n^{2}-n\right)$.

\subsection{Related Work}

It is empirically known that the diameter and ASPL of a random graph are smaller than those obtained by regularly connecting edges with vertices. Research has thus been conducted to utilize the randomness in a random graph for various networks $[1,7,10,18]$. In most cases, however, the diameter and ASPL of random graphs are larger than the respective lower bounds. Thus, it is important to find a graph with the smallest possible diameter and ASPL.

The Graph Golf official website[13] links to several slides and papers by past participants[15, 16, 19]. Existing methods can be classified into the following two categories. (1) Multiple graphs with few vertices are combined to create a target graph[16, 19]. Although this method requires almost no computation for the APSP problem, only graphs with specific numbers of vertices and degrees can be created. (2) Based on a random graph, a target graph with the smallest diameter and ASPL is created using an optimization method such as SA[15]. Although this method can create graphs with arbitrary numbers of vertices and degrees, since an enormous number of calculations for the APSP problem is necessary, approximation is often used. Since errors are generated using approximation, there is a possibility that the solution search performance of the optimization method may be adversely affected. Moreover, the approximation itself may have constraints depending on a target graph. For example, [15] supports only a graph having a diameter 3.

The method proposed in the present study creates a graph by combining multiple graphs with few vertices symmetrically, and optimizes it using SA while maintaining its symmetry. In addition, in order to find accurate solutions with SA, the method does not approximate. The proposed method thus combines the features of the two categories mentioned above. However, there are little constraints on the numbers of vertices and degrees, as described in Section 3.3.6. In addition, the calculation time required for the APSP problem is drastically reduced by using graph symmetry and hybrid parallelization with MPI and OpenMP.

\section{SIMULATED ANNEALING BASED ON GRAPH SYMMETRY}

Section 3.1 gives an overview of SA, Section 3.2 applies SA to ODPs, and Section 3.3 describes the proposed method based on SA.

\subsection{Overview of Simulated Annealing}

SA is a general purpose optimization algorithm that simulates the physical process of heating a material and then slowly lowering its temperature. SA searches not only in the direction in which the solution is improved, as done by the hill climbing method, but also conducts a probabilistic search in the direction in which the solution gets worse. With this feature, SA can escape from local optima to find the most efficient solution. In addition, compared 


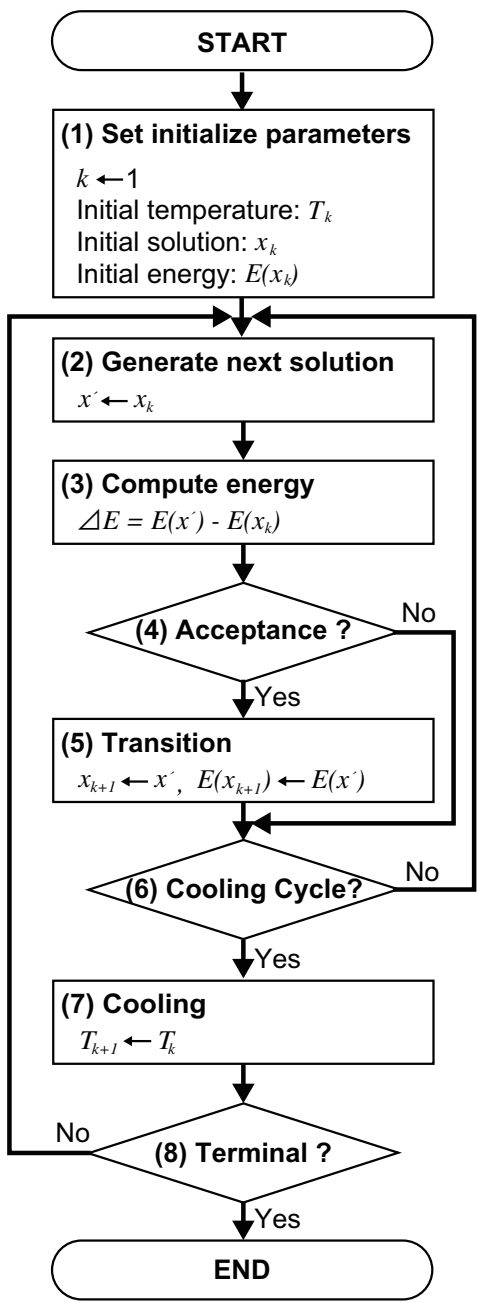

Figure 2: Algorithm of simulated annealing

with other evolutionary calculation methods such as genetic algorithms (GAs) [4, 8], SA is much simpler and more versatile, and thus easier to apply to practical problems.

SA has a parameter called temperature. As the solution search progresses, the temperature changes from a high state to a low state. Fig. 2 shows the algorithm of SA. (1) Set the value $k=1$, an initial temperature $T_{k}$, and initial solution $x_{k}$. Then, energy $E\left(x_{k}\right)$, corresponding to $x_{k}$, is calculated (the lower the energy, the better). (2) Generate new solution $x^{\prime}$ based on $x_{k}$. (3) Calculate $E\left(x^{\prime}\right)$, and obtain energy difference $\Delta E=E\left(x^{\prime}\right)-E\left(x_{k}\right)$. If $E\left(x^{\prime}\right)$ is the optimal solution, the solution search is terminated (not shown in Fig. 2). (4) Decide whether to accept new solution $x^{\prime}$ according to the energy difference $\Delta E$ and temperature $T_{k}$. (5) In the new solution is accepted, change $x^{\prime}$ to $x_{k+1}$ and $E\left(x^{\prime}\right)$ to $E\left(x_{k+1}\right)$. (6) Repeat steps (2) to (5) a certain number of times. (7) Change temperature $T_{k}$ to new temperature $T_{k+1}$. (8) Terminate SA when the temperature drops sufficiently or when it reaches another certain number of times.
For judging the solution in step (4) in Fig. 2, the following Metropolis criterion is often used.

$$
\text { Probability }=\left\{\begin{array}{lr}
1 & \text { if } \Delta E<0 \\
\exp \left(-\frac{\Delta E}{T}\right) & \text { otherwise }
\end{array}\right.
$$

With the Metropolis criterion, if the energy of the new solution is better than that of the current solution, the new solution is accepted; otherwise, the new solution is probabilistically accepted. The higher the temperature is, the higher is its acceptance probability. In other words, a global search is carried out positively in the direction of worse solutions in the early stage of the solution search, and a local search is carried out in the direction of better solutions at the end of the solution search.

In step (7) in Fig. 2, the following exponential annealing is often used.

$$
T_{k+1}=\alpha T_{k} \quad(\alpha \leq 1)
$$

New temperature $T_{k+1}$ is calculated by multiplying current temperature $T_{k}$ by cooling rate $\alpha$. As the value of $\alpha$ gets closer to 1.0, the cooling speed gets slower, so the probability of finding a good solution increases but the number of iterations until the temperature decreases increases.

\subsection{Simulated Annealing for Order/Degree Problem}

3.2.1 Generation of Initial Solution. In order to generate an initial solution, the method random_regular_graph() of the Python package networkx[14] for graph theory is used ${ }^{2}$. This method outputs a regular graph with the specified numbers of vertices and degrees. Moreover, the output regular graph is a simple graph (i.e., it contains no loops or multiple edges).

3.2.2 Energy Calculation Equation. Since solving an ODP requires finding the smallest diameter and ASPL, it is natural to use the diameter and ASPL in the energy calculation equation. With the diameter at a certain solution $x$ denoted as $D(x)$, ASPL denoted as $\operatorname{ASPL}(x)$, and the weight denoted as $w$, the value of energy $E(x)$ can be expressed by the following equation, where $w$ is an appropriate positive real number.

$$
E(x)=w \times D(x)+\operatorname{ASPL}(x)
$$

In this equation, it is necessary to set $w$ for each problem in an ODP. Such parameter tuning is time-consuming. Preliminary experiments showed that if $w$ is assigned a large value, the search performance deteriorates because it is difficult to search in the direction of worse solutions. Since the network topology with the smallest ASPL is considered to also have the smallest diameter, for the proposed method, $w$ is set to 0 in the above equation and the diameter is not used in the energy calculation equation.

3.2.3 Generation of New Solution. In SA, the next solution is generated by adding a small perturbation to the current solution. The perturbation range is called the neighborhood. For combinatorial optimization problems such as ODPs, the range in which a solution can be changed with the minimum width is defined as the neighborhood.

\footnotetext{
${ }^{2}$ The Python program using this method is available on the official website of Graph Golf. http://research.nii.ac.jp/graphgolf/py/create-random.py
} 


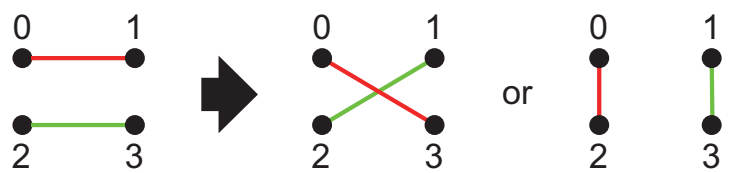

Figure 3: Overview of 2-opt method

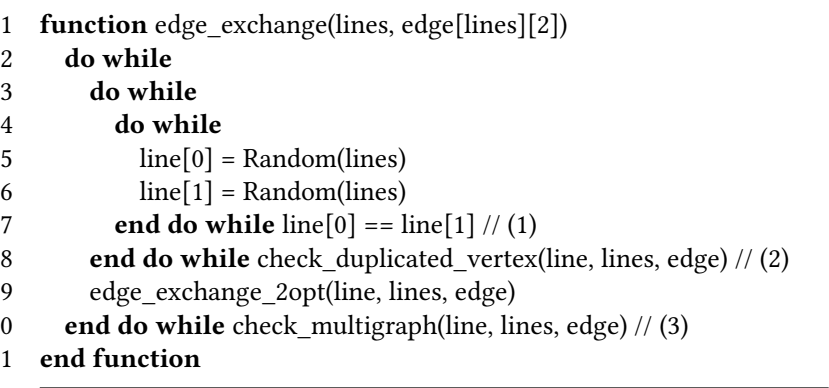

Figure 4: Pseudo-code for generation of new solution

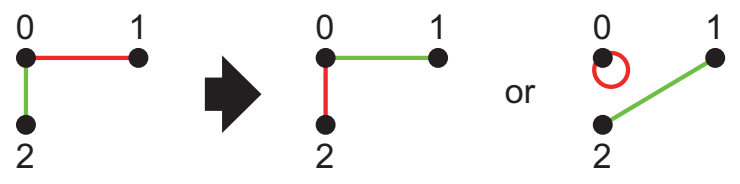

Figure 5: One of the vertices of two edges is the same

The proposed method generates the next solution using the 2opt method, which is often used for the traveling salesman problem. Fig. 3 shows an overview of the 2-opt method, where two edges are randomly selected from the graph, and the next solution is generated by exchanging them. There are two ways of exchanging edges, one of which is chosen randomly.

Fig. 4 shows the pseudo-code for the 2-opt method. In line 1, the variable lines is the total number of edges, and the array edge stores information on edges. The second dimension of edge indicates both ends of the edge. In lines 5-6, two edges are randomly selected from all the edges. For the following cases, the selection of edges is redone. (1) Two selected edges are the same. (2) One of the vertices of the selected two edges is the same. As shown in Fig. 5 , even if these lines are exchanged, the same graph or a loop is generated, and thus no improvement in energy is expected. (3) A graph with multiple edges is generated after the 2-opt method is applied. The energy of such a graph is considered to be worse than that of a simple graph. (4) A disconnected graph is generated after the 2-opt method is applied. (not shown in Fig. 4). Since it is possible to evaluate the connectivity of a graph by performing BFS, the proposed method evaluates connectivity as it calculates the ASPL after the function edge_exchange().

3.2.4 Calculation of Energy Difference. According to the definitions of the distance matrix and ASPL given in Section 2.1, the minimum value of the energy difference $\Delta E$ used in equation (5) is $2 /\left(n^{2}-n\right)$. That is, as the number of vertices increases, the minimum value of $\Delta E$ decreases. Since the setting of the temperature

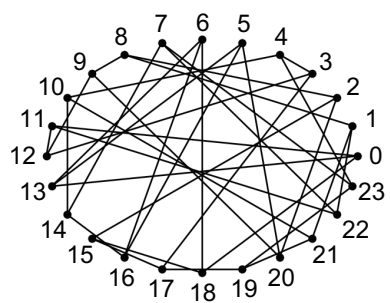

(a) $\mathrm{g}=1$

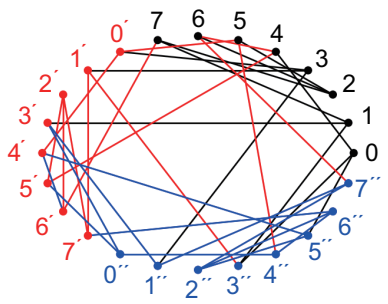

(c) $g=3$

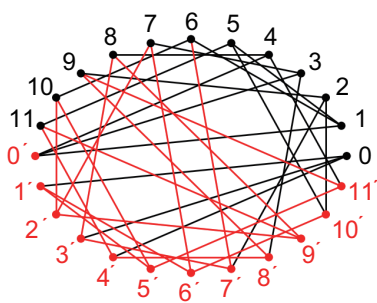

(b) $\mathrm{g}=2$

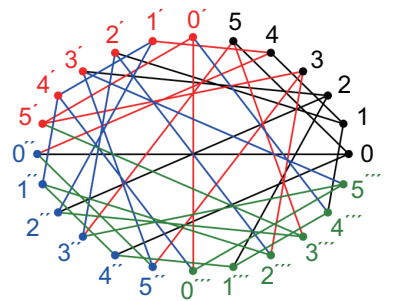

(d) $g=4$
Figure 6: Examples of graph symmetry with $n=24, d=3$

parameter becomes complicated if its minimum value is different for each problem in the ODP, the energy difference $\Delta E$ is multiplied by $\left(n^{2}-n\right)$ as the weight as follows.

$$
\Delta E=\left(E\left(x^{\prime}\right)-E\left(x_{k}\right)\right) \times\left(n^{2}-n\right)
$$

This equation means that the minimum $\Delta E$ is 2 for all problems.

\subsection{Proposed Method}

3.3.1 Overview. As mentioned in Section 1, finding the best solution for an ODP is difficult because the search space is very wide and it takes a long time to calculate the ASPL. By giving symmetry to the graph, the propose method reduces both the search space and the calculation time of the ASPL.

Fig. 6 shows examples of the graph symmetry used in the proposed method. Each graph has $n=24, d=3$, and a symmetrical topology. The variable $g$ in the figure is the number of groups. When a graph is viewed as a plane, if it is rotated by $360 / g$ degrees, the connection relationship between the edge and the vertex becomes the same graph. For the case of $g=1$, a normal graph (not symmetrical) is obtained.

3.3.2 Generation of Initial Solution. Before the solution search is started, an initial solution with a symmetrical topology must be generated. This section explains how to generate the initial solution using the method random_regular_graph() described in Section 3.2.1.

Fig. 7 shows an outline of how to generate an initial solution for the graph in Fig. $6 \mathrm{~d}$ with $n=24, d=3$, and $g=4$. (1) Create a graph using the method random_regular_graph() with the number of vertices of the target graph divided by $g(6=n / g)$. The number of degrees of the graph is the same as that for the target graph. This graph is called the base graph. After duplicating $g$ base graphs, select one edge from each base graph so that it becomes symmetrical. For convenience, the four base graphs are rotated by 90 degrees in 
(1)
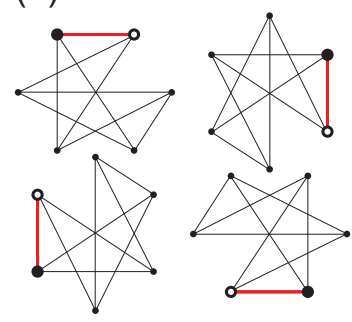

(2)

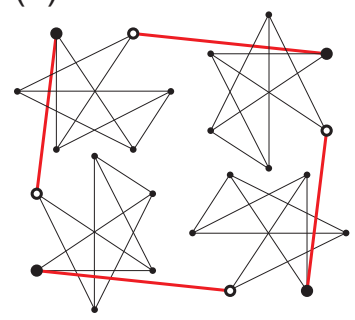

Figure 7: Initial graph $(r=1)$

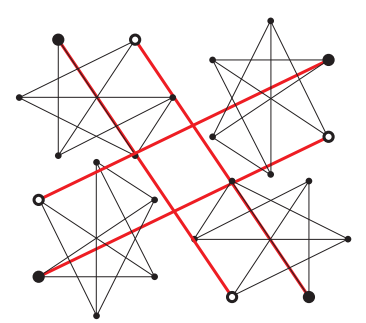

(a) $r=2$

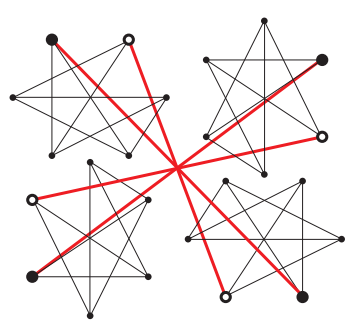

(b) Special case for even $g$
Figure 8: Failure examples of generation of initial solution

Fig. 7, and the starting point $\circ$ and the end point $\bullet$ of the selected edge are set. (2) Connect the start point $\circ$ and the end point $\bullet$ of the base graph separated by $r$ in the clockwise direction. Fig. 7 shows the case for $r=1$ and $r$ as a random integer value from 1 to $g-1$.

Fig. 8 shows failure examples of edge exchange. These graphs are disconnected; they have unconnected partial structures (the base graphs on the upper left and the upper right are not connected). In an ODP, a disconnected graph cannot be treated as a solution. Fig. 8a shows the case of $r=2$.

Fig. 8b shows another edge exchange method, different from that in Fig. 7. If the value of $g$ is an even number, graphs can be created by connecting the start points and the end points of the base graphs in the direction rotated 180 degrees. The solution obtained by this operation is in the neighborhood of the current solution and is important for generating a new solution, as described in the next section. However, when this operation is performed on the initial base graph, the graph becomes disconnected.

As described above, when a disconnected graph is obtained after exchanging edges, edges and $r$ are selected again to repeat the edge exchange until a connected graph is obtained. This edge exchange method is called the $1 \mathrm{~g}$-opt method in this paper. The $1 \mathrm{~g}$ opt method is also used to generate new solutions.

3.3.3 Generation of Next Solution. The proposed method generates a new solution in the neighborhood of the current solution while maintaining graph symmetry. This operation uses the $1 \mathrm{~g}$ opt method and the 2g-opt method, extend from the 2-opt method described in Section 3.2.3. Fig. 9 shows the pseudo-code for creating a new solution using this operation. This code is an extension of Fig. 4; the main changes are in lines 9-13. The variable groups means the number of groups.

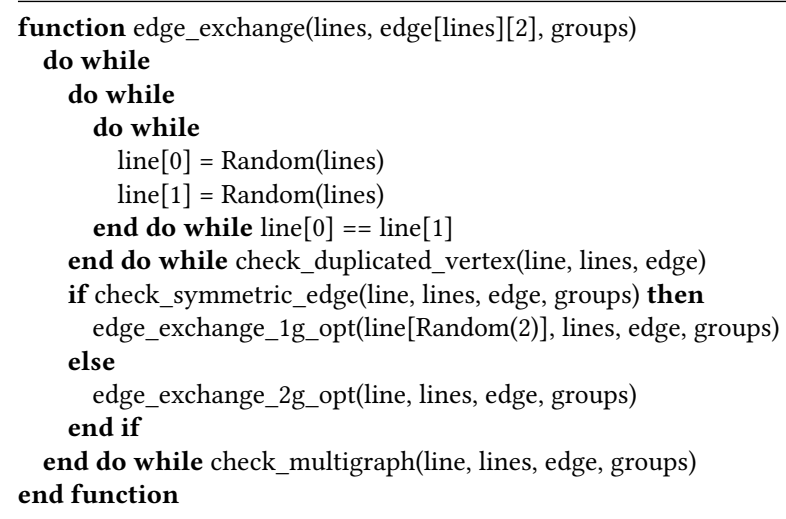

Figure 9: Pseudo-code for generation of new symmetrical solution
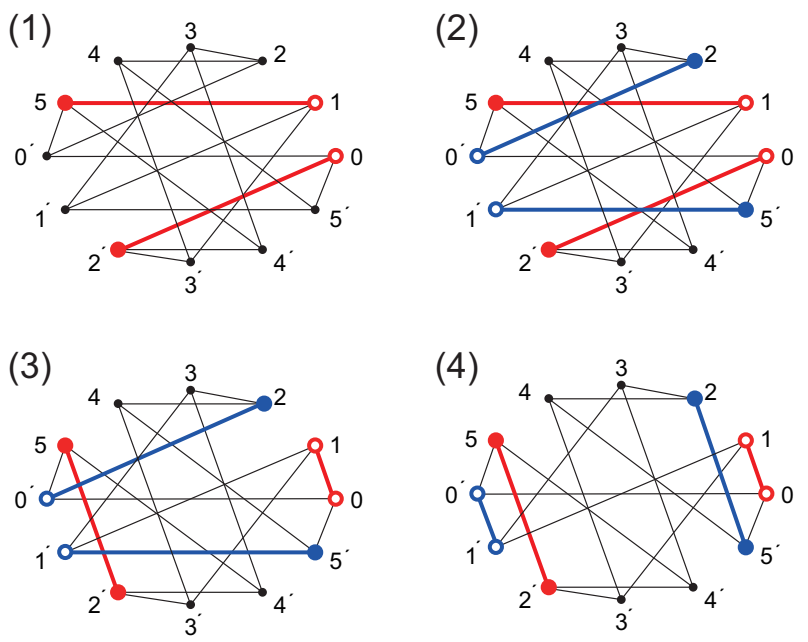

Figure 10: Overview of $2 \mathrm{~g}$-opt method $(n=12, d=3, g=2)$

Fig. 10 shows an outline of the $2 \mathrm{~g}$-opt method executed in line 12 in Fig. 9. Fig. 10 uses graphs with $n=12, d=3$, and $g=2$. (1) Randomly select two edges from all the edges. (2) Select edges symmetrically related to the selected two edges. (3) Apply the 2opt method to the edges selected in (1). (4) Apply the 2-opt method to the edges selected in (2) in the same way as (3).

Fig. 11 shows failure examples of the above $2 \mathrm{~g}$-opt method. Fig. 11a shows that one edge (or both edges) selected in (1) of Fig. 10 is symmetrical. Fig. 11b shows that the two edges selected in (1) of Fig. 10 are symmetrical. For the above cases, it is impossible to perform operation (2) of Fig. 10. If the $2 \mathrm{~g}$-opt method cannot be applied, the $1 \mathrm{~g}$-opt method is used instead, as shown in lines 9-10 of Fig. 9. Specifically, one edge is randomly selected from the two selected edges, and the $1 \mathrm{~g}$-opt method is applied to it.

3.3.4 Reduction of Calculations for ASPL. By using the symmetry of the graph, it is possible to reduce the calculations required for the ASPL. In general, it is necessary to calculate the distance from all vertices to all other vertices using BFS. However, with graph 


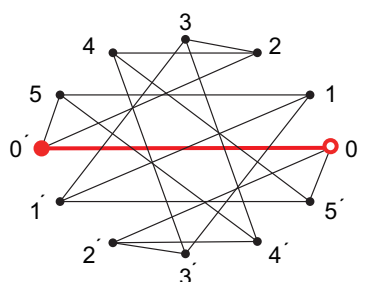

(a) One selected edge is symmetrical

Figure 11: Failure examples of $2 \mathrm{~g}$-opt method

symmetry, the distances from the vertex to all other vertices are the same for all symmetrically related vertices. For example, in Fig. $6 \mathrm{~d}$, from vertices $0,0^{\prime}, 0^{\prime \prime}$, and $0^{\prime \prime \prime}$, the sets of distances to all other vertices are the same. That is, it is sufficient to perform BFS from $(n / g)$ number of vertices. In Fig. 6d, the ASPL can be calculated by merely performing BFS from vertex 0 to vertex 5 .

As mentioned in Section 1, the calculation complexity for the ASPL is $O\left(n^{2} d\right)$; by applying this calculation reduction method, the complexity becomes $O\left(n^{2} d / g\right)$.

3.3.5 Calculation of Energy Difference. Compared with the 2-opt method, the symmetrical edge exchange method described in Section 3.3.3 performs more edge exchanges. Therefore, the energy difference $\Delta E$ in equation (8) increases. Preliminary experiments showed that the change amount increased in proportion to the number of groups $g$.

When solving a certain problem in an ODP using the proposed method, it is cumbersome to adjust the temperature parameter each time $g$ is changed. Therefore, in order to enable solution search at the same temperature regardless of $g$ for a given problem, the equation is multiplied by $1 / g$.

$$
\Delta E=\left(E\left(x^{\prime}\right)-E\left(x_{k}\right)\right) \times\left(n^{2}-n\right) / g
$$

3.3.6 Constraint Condition. The constraints of the proposed method are as follows. (1) Because the graph has a symmetrical topology, the number of groups $g$ needs to be a divisor of the number of vertices $n$. (2) Since the vertex number is always larger than the number of degrees in the regular graph, the base graph needs to have $n / g>d$. However, after creating a graph using the $1 \mathrm{~g}$-opt method with an appropriate value of $d$, symmetrical edges can be manually added to each vertex (i.e, $d$ is increased) to avoid the constraint (2).

\section{EVALUATION OF SOLUTION SEARCH PERFORMANCE}

\subsection{Parameter Setting Guidelines}

The SA parameters shown in Section 3.1 are initial temperature (maximum temperature) $T$, end temperature (minimum temperature) $C$, number of repetitions for the cooling process $I$, and total number of calculations $N$. The cooling rate $\alpha$ in equation (6) is derived from the above four values using the following equation.

$$
\alpha=(C / T)^{I / N}
$$

The guidelines for each parameter are given below.
- For $T$, it is preferable for the acceptance probability of the solution in the direction of deterioration in the early stage of the solution search to be high. Therefore, 100 new solutions are generated from a random graph and the maximum energy difference $\Delta E$ is stored. Next, $T$ is set so that the maximum $\Delta E$ is accepted with a probability of $50 \%$ in equation (5).

- For $C$, it is preferable for the acceptance probability of the solution in the direction of deterioration at the end of the solution search to be low. As mentioned in Section 3.2.4, the minimum $\Delta E$ for all problems is 2 . Therefore, $C$ is set so that the minimum $\Delta E$ is accepted with a probability of $0.01 \%$ in equation (5). The value is always 0.22 .

- For $I$, preliminary experiments showed that $I$ does not affect solution search performance. Therefore, $I$ is set to 1 .

- For $N$, which is proportional to the calculation time, is set according to the time and calculation resources of the user. In this paper, we set $N$ to $10^{7}$.

In the proposed method, the number of groups $g$ is added to the parameters mentioned above. Section 4.2 investigates the relationship between $g$ and solution search performance.

\subsection{Solution Search Performance}

In order to evaluate the solution search performance of the proposed method, three problems, $(n, d)=(72,4),(256,5),(256,10)$, are used from among the problems provided by Graph Golf in 2018[13]. $T$ values for the three problems are 238.91, 452.43, and 79.63, respectively.

For each problem, the proposed method was executed 100 times with different numbers of groups $g$. Fig. 12 shows the results, where "ASPL Gap" on the vertical axis is the difference between the final value obtained by the proposed method and the lower bound $L_{n, d}$ of the ASPL. Fig. 12 shows the worst, average, and best values of ASPL Gap. These results show that the solution search performance tends to increase as the number of groups $g$ increases. However, in Fig. 12a, the solution search performance is better for $g=9$ than for $g=12$, indicating that solution search performance may deteriorate if the value of $g$ is too large. The pair of each problem and $g$ where the average value of ASPL Gap was the best was $(n, d, g)=(72,4,9),(256,5,32),(256,10,16)$.

The solution search performance degraded when $g$ was large for the following reason. $g$ expresses the strength of regularity of a graph; regularity becomes stronger as $g$ increases. As mentioned in Section 2.2, the ASPL of a random graph tends to be shorter than that of a regular graph. Thus, regularity becomes too strong because $g$ is too large, deteriorating search performance.

\subsection{Fixed-Temperature Simulated Annealing}

As shown in the previous section, the temperature in SA changes from high to low. It has been reported that solution search performance may be improved by using a fixed temperature from the start of the solution search to the end[5]. Since the maximum temperature $T$ and the minimum temperature $C$ are the same value in fixed-temperature $\mathrm{SA}$, the value of the cooling rate $\alpha$ in equation 


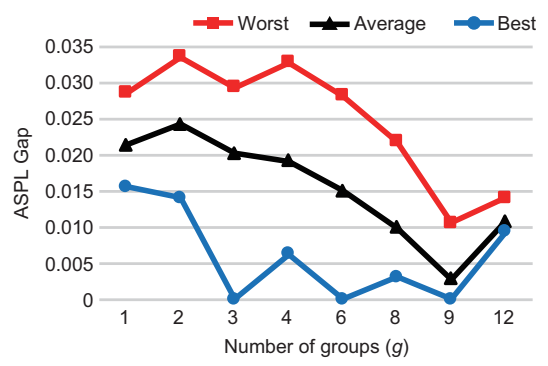

(a) $n=72, d=5$

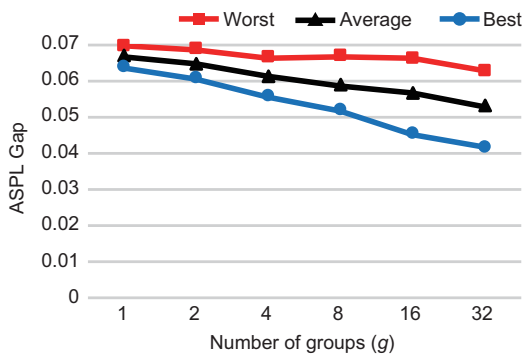

(b) $n=256, d=5$

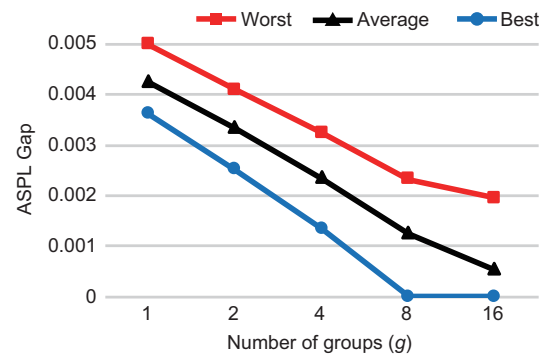

(c) $n=256, d=10$

Figure 12: Results of solution search performance evaluation

Table 1: Comparison of normal and fixed-temperature simulated annealing

\begin{tabular}{c|r|r|r|r}
\hline \hline \multirow{2}{*}{$(n, d, g)$} & \multicolumn{2}{|c|}{ Optimum solutions } & \multicolumn{2}{c}{ Calculations } \\
\cline { 2 - 5 } & $\begin{array}{r}\text { Fixed } \\
\text { Temp. }\end{array}$ & Normal & $\begin{array}{r}\text { Fixed } \\
\text { Temp. }\end{array}$ & Normal \\
\hline$(72,4,9)$ & $100 / 100$ & $70 / 100$ & 92,564 & 621,272 \\
$(256,10,16)$ & $77 / 100$ & $17 / 100$ & 442,372 & 921,633 \\
\hline
\end{tabular}

(10) is always 1 . This section compares the solution search performance of fixed-temperature SA with that of normal SA using the problems described in the previous section.

For fixed-temperature SA, the $T$ and $C$ set in the previous section were geometrically divided into 100 pieces. Fixed-temperature SA was then executed 100 times at each temperature. The other parameters were the same as those in the previous section. Fig. 13 shows the results where while $n=0$ on the horizontal axis is $T$, $n=99$ is $C$. The graph below shows the enlargement of the temperature around the best temperature for the average solution. In the graph, the dotted lines are the worst, average, and best values obtained with the optimal parameters for normal SA in Fig. 12. Fig. 13 confirms that fixed-temperature SA has higher solution search performance if the temperature parameter $n$ is optimal and around it.

In addition, as other metrics for solution search performance, Table 1 shows the number of optimal solution discoveries and the average number of energy calculations required for finding the optima for the problems $(n, d, g)=(72,4,9)$ and $(256,10,16)$ (for which optima were found). The result for fixed-temperature SA is the result obtained with the temperature with the best average value. Table 1 confirms that fixed-temperature SA produces more optimal solutions and required fewer energy calculations.

The temperature at which solution search performance becomes strong for fixed-temperature SA is between $T$ and $C$. From Fig. 13, the relationship between solution search performance and temperature can be regarded as a relatively simple unimodal problem. By using a simple optimization method, it is possible to determine the temperature at which good performance can be achieved. The specifics will be investigated in future work.
Table 2: Specifications of COMA system

\begin{tabular}{l|l}
\hline \hline CPU & Intel Xeon-E5 2670v2, 10 Cores $\times$ 2 Sockets \\
Memory & DDR3-SDRAM, 1866MHz, 59.7GB/s, 64GB \\
Network & InfiniBand FDR, 7GB/s \\
Software & intel/18.0.1, intelmpi/2018.1 \\
\hline
\end{tabular}

\section{EVALUATION OF HIGH PERFORMANCE}

\subsection{Use of Graph Symmetry}

As described in Section 3.3.4, the calculation of the ASPL can be reduced by using graph symmetry. This section examines the relationship between the number of groups $g$ and the calculation time of the ASPL using the problems $(n, d)=(72,4),(256,5)$, and $(256,10)$. The BFS algorithm used to calculate the ASPL is described in Section 5.2. For this evaluation, the COMA system, located at the University of Tsukuba, Japan, was used. Its specifications are shown in Table 2. The optimization option used for the compiler was "O2”. The program was executed as one process and one thread on a single compute node in this section.

Fig. 14 shows the calculation time for 100 ASPLs for each problem. The bar graph shows the time on the left vertical axis, and the line graph shows the speed up ratio with $g=1$ on the right vertical axis. Speed ups of 8.11, 31.76, 15.67 times, respectively, were achieved for $(N, d, g)=(72,4,12),(256,5,32)$, and $(256,10,16)$. The performance for the problem $(72,4,12)$ is not scaled somewhat because the time required for processing such as initialization irrelevant to $g$ is affected because BFS time per search is short compared that for the other problems.

\subsection{Hybrid Parallelization with MPI and OpenMP}

This section describes hybrid parallelization with MPI and OpenMP for APSL calculation in the proposed method and evaluates its performance. For the hybrid parallelization, multiple BFS are simultaneously executed using MPI, and each BFS is executed in parallel using several OpenMP threads. As mentioned in Section 3.3.4, the calculation complexity for the ASPL is $O\left(n^{2} d / g\right)$; by applying this calculation reduction method, the complexity becomes $O\left(n^{2} d /(g P T)\right)$ when the number of MPI ranks is $P$ and the number of OpenMP threads is $T$. 

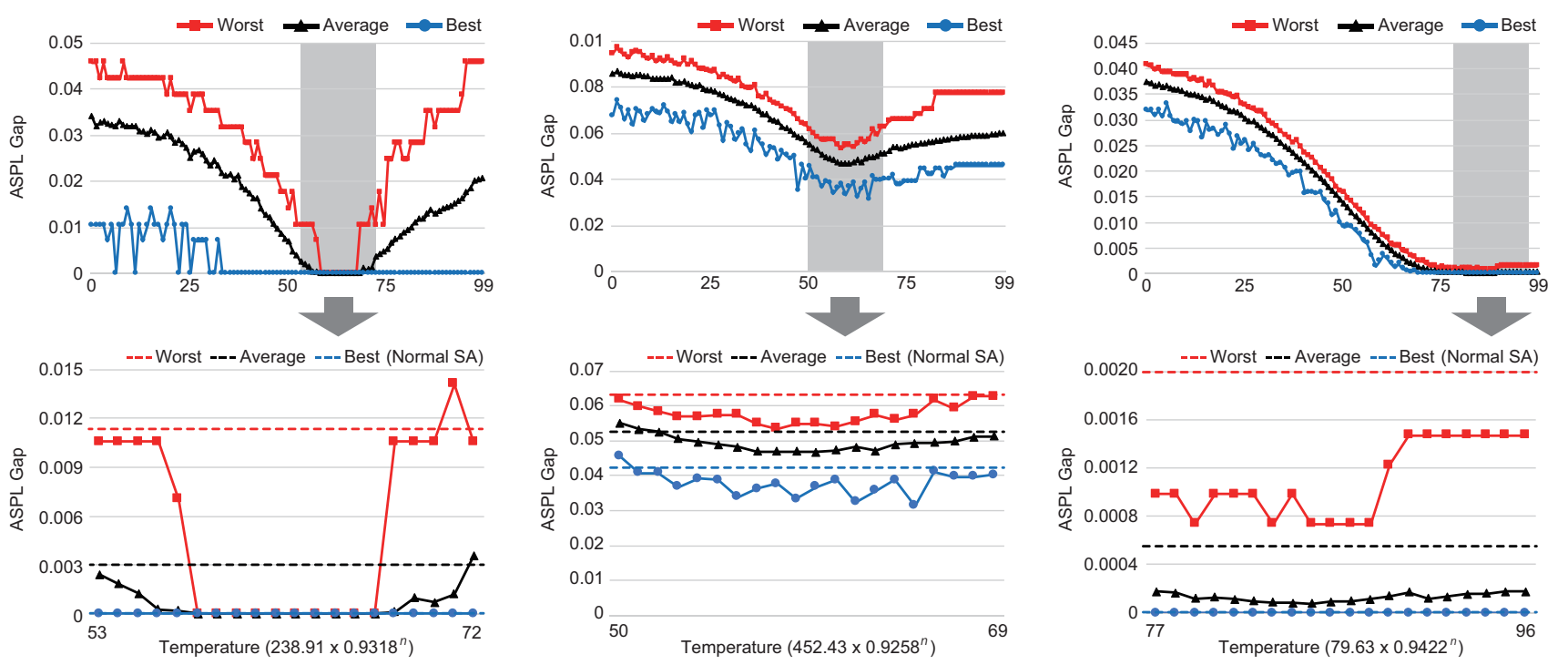

(a) $n=72, d=4, g=9$

(b) $n=256, d=5, g=32$

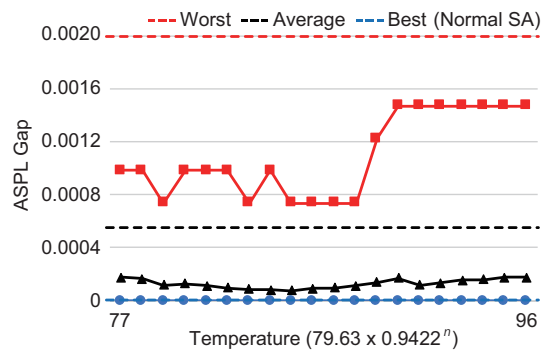

(c) $n=256, d=10, g=16$

Figure 13: Solution search performance for fixed-temperature simulated annealing

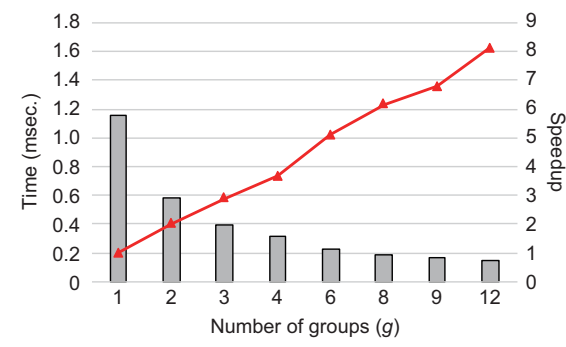

(a) $n=72, d=4$

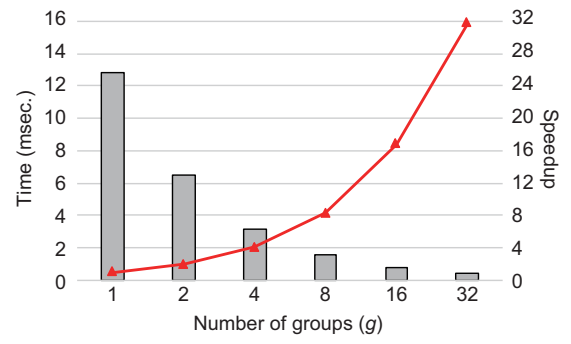

(b) $n=256, d=5$

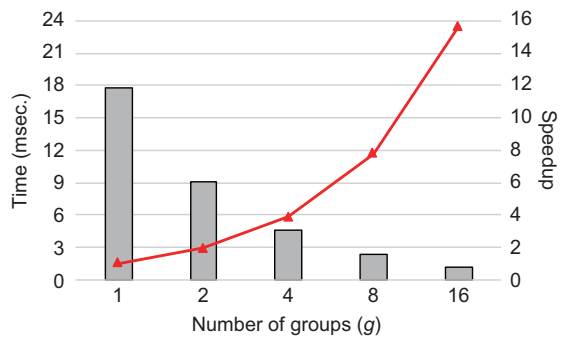

(c) $n=256, d=10$

Figure 14: Relationship between the number of groups $g$ and calculation time for ASPL

As described in Section 3.3.4, it is necessary to perform BFS from $(n / g)$ vertices to calculate the ASPL. Since BFS can be executed in parallel, the proposed method assigns the starting vertices to MPI ranks. Each MPI rank performs BFS to calculate the distance between its vertex and all other vertices, and aggregates the total distance using the function MPI_Allreduce(). Therefore, the upper limit of the number of MPI ranks is $(n / g)$. In addition, the method checks the connectivity of the target graph while executing BFS, and the results are aggregated using the function MPI_Allreduce().

The proposed method uses the top-down approach of level synchronized BFS[2]. The pseudo-code is shown in Fig. 15. Furthermore, Fig. 16 shows the BFS pseudo-code using thread parallelization based on lines 12-21 of Fig. 15. The level-synchronized BFS repeats the process of finding the set of next vertices next by tracing one edge from a current vertex set frontier and setting it as the input of the next process. In line 3 in Fig. 16, each thread splits the frontier, which is the current starting point set. In line 6, it is checked whether the next search point has already been visited. If not, the distance from the starting point is assigned to parents. This operation requires thread barrier synchronization; specifically, it is implemented using the function__sync_bool_compare_and_swap(). In lines 10-13, local_next, which is a set of newly visited vertices found by each thread, is stored in next.

For the proposed method with hybrid parallelization, the time required for ASPL calculation was measured. The measurement environment was the same as that in the previous section. With this hybrid parallelization, its efficiency is expected when the problem size is large. Thus, the largest problem $(n, d)=(400000,32)$ in Graph Golf 2018 was used for evaluation. The number of groups $g$ was set to 10000 . Therefore, the maximum number of MPI ranks was 40. A single CPU socket was assigned to one MPI rank. Since each computing node in the COMA system has two CPU sockets, 20 computing nodes were used. For thread parallelization, up to 10 OpenMP threads were assigned per CPU socket.

Fig. 17 shows the calculation time for 100 ASPLs. Fig. 17a shows the results obtained using only OpenMP thread parallelization within a single CPU socket, and Fig. 17b shows the results obtained using hybrid parallelization with MPI and OpenMP. In Fig. 17a, the performance obtained with $1 P 10 T$ was 5.97 times higher than that obtained with $1 P 1 T$. Since barrier synchronization was performed, 


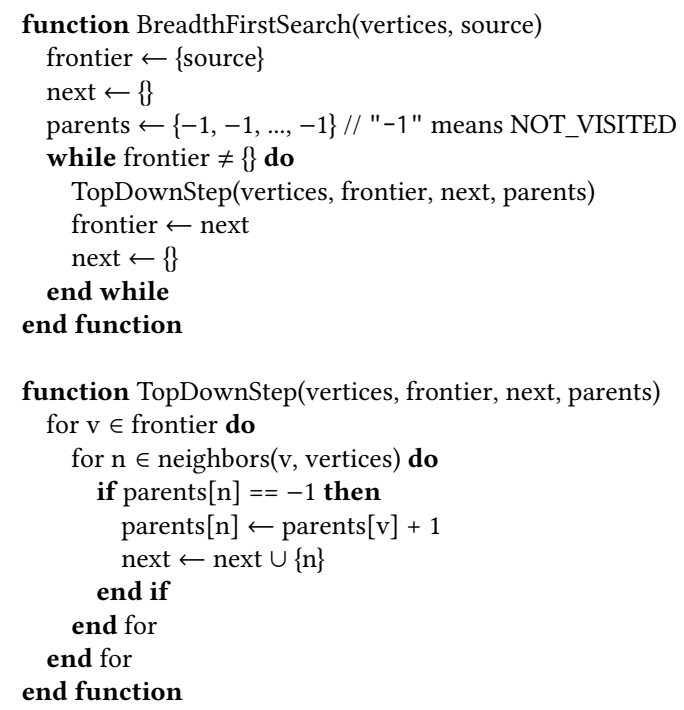

Figure 15: Pseudo-code for breadth-first search

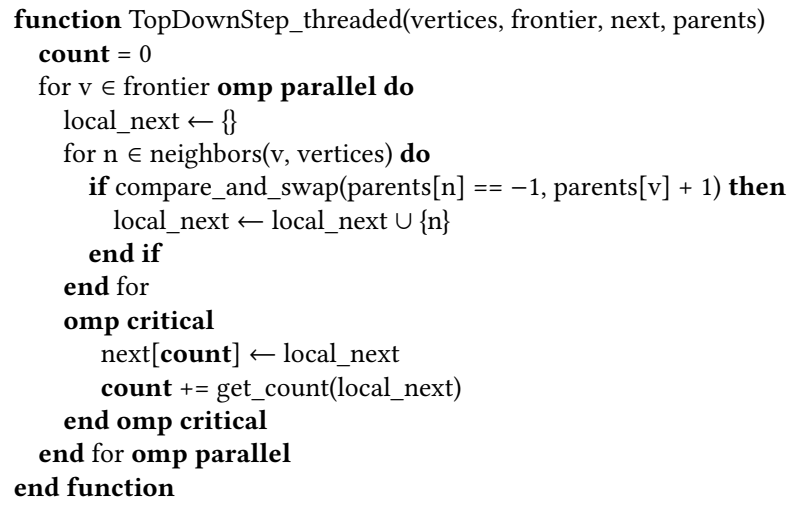

Figure 16: Pseudo-code for threaded breadth-first search

the performance does not perfectly scale with the number of threads. In Fig. 17b, the performance obtained with $40 P 10 T$ is 35.12 times higher than that obtained with $1 P 10 T$. Since the communication used in the method only carries out one element MPI_Allreduce() twice, the ratio of communication time to total calculation time is small. Therefore, parallelization efficiency with MPI is very high. Finally, the performance obtained with $40 P 10 T$ is $209.80(=5.9744 \times$ 35.1161) times higher than that obtained with $1 P 1 T$. In addition, since graph symmetry was also applied, the performance improvement was about 2,098,000 times compared to that obtained with $g$ $=1$ and $1 P 1 \mathrm{~T}$.

\section{SUMMARY AND FUTURE WORK}

This study developed a method for solving ODPs based on SA to produce a network topology with low latency. In the proposed method, the topology is made symmetrical, making it possible to efficiently find a good solution. The proposed method was applied

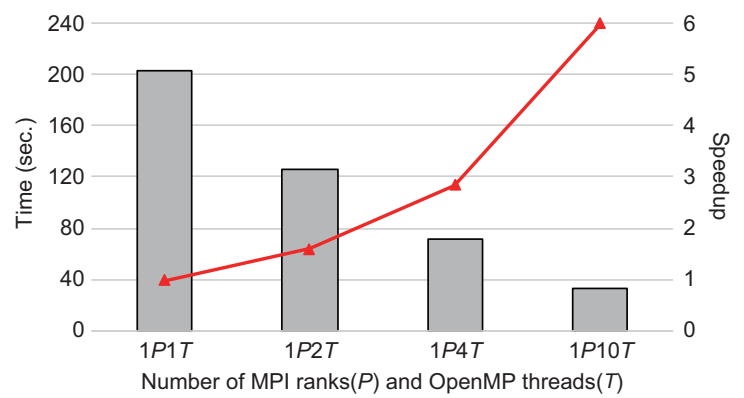

(a) Thread parallelization with OpenMP

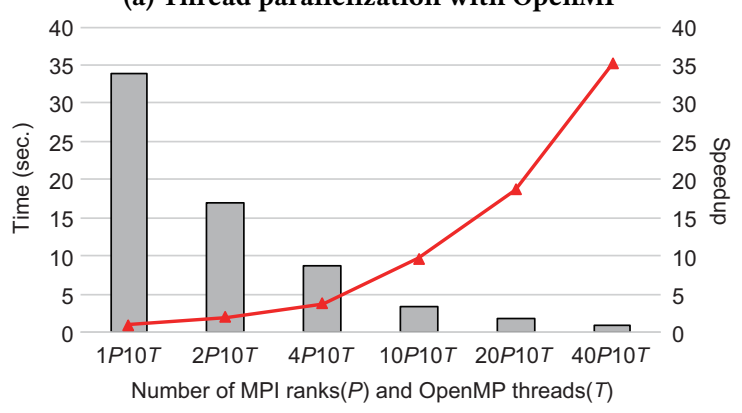

(b) Hybrid parallelization with MPI and OpenMP

Figure 17: Result of high speed $(n=400000, d=32, g=10000)$

to the problems $(n, d)=(72,4),(256,5),(256,10)$ provided by Graph Golf 2018. For problems $(72,4)$ and $(256,10)$, the optimal solutions were found. The network topology had a sufficiently small ASPL, even for the problem $(256,5)$. Moreover, making the topology of the graph symmetrical reduced the calculation time required for the ASPL. As a result, the proposed method achieved speed ups of $8.11,31.76$, and 15.67 times, respectively, for problems $(72,4)$, $(256,5)$, and $(256,10)$. Furthermore, by utilizing hybrid parallelization with MPI and OpenMP, the calculation time for the ASPL was further reduced. A performance improvement of 209.80 times was achieved for the problem $(n, d)=(400000,32)$. When symmetry and parallelization were combined, even faster performance was achieved.

Future work will focus on the following. (1) We will evaluate the solution search performance of the proposed method for other problems provided by Graph Golf. (2) The proposed method may not have sufficient performance when the number of vertices is a prime number because the number of groups $g$ will be 1 . In order to overcome this issue, the method will be extended to obtain symmetry even if the number of vertices is prime. (3) As mentioned in Section 4.3, we will develop a method for automatically finding the optimal temperature for fixed-temperature SA. (4) We will compare the solution search performance of SA with that of other evolutionary calculation methods such as GA. In particular, since GA uses crossover, it should be effective for problems with partial solutions, such as ODPs. (5) For further speed ups, we will use an accelerator such as a graphical processing unit. Moreover, in the proposed method, parallelization for one BFS uses only OpenMP thread parallelization. Thus, further speed ups can be realized by performing one BFS using hybrid parallelization with MPI and OpenMP. 


\section{ACKNOWLEDGEMENTS}

This research used the coma system provided by Interdisciplinary Computational Science Program in the Center for Computational Sciences, University of Tsukuba. And this research was supported by JSPS KAKENHI Grant Number 18K11331.

\section{REFERENCES}

[1] Ankit Singla and Chi-Yao Hong and Lucian Popa and Philip Brighten Godfrey. 2011. Jellyfish: Networking Data Centers Randomly. CoRR abs/1110.1687 (2011). arXiv:1110.1687 http://arxiv.org/abs/1110.1687

[2] Scott Beamer, Krste Asanović, and David Patterson. 2012. Direction-optimizing Breadth-first Search. In Proceedings of the International Conference on High Performance Computing, Networking, Storage and Analysis (SC '12). IEEE Computer Society Press, Los Alamitos, CA, USA, Article 12, 10 pages.

[3] V. G. Cerf, D. D. Cowan, R. C. Mullin, and R. G. Stanton. 1974. A lower bound on the average shortest path length in regular graphs. Networks 4, 4 (1974), 335-342. https://doi.org/10.1002/net.3230040405

[4] David E. Goldberg. 1989. Genetic Algorithms in Search Optimization and Machine Learning. AI Magazine 12 (1989), 102-103.

[5] Fielding, M. 2000. Simulated Annealing With An Optimal Fixed Temperature. SIAM fournal on Optimization 11, 2 (2000), 289-307.

[6] Floyd, Robert W. 1962. Algorithm 97: Shortest Path. Commun. ACM 5, 6 (June 1962), 345-. https://doi.org/10.1145/367766.368168

[7] H. Matsutani and M. Koibuchi and I. Fujiwara and T. Kagami and Y. Take and T. Kuroda and P. Bogdan and R. Marculescu and H. Amano. 2014. Low-latency wireless 3D NoCs via randomized shortcut chips. In 2014 Design, Automation Test in Europe Conference Exhibition (DATE). 1-6. https://doi.org/10.7873/DATE.2014.286

[8] John H. Holland. 1992. Adaptation in Natural and Artificial Systems: An Introductory Analysis with Applications to Biology, Control and Artificial Intelligence. MIT Press, Cambridge, MA, USA.

[9] S. Kirkpatrick, C. D. Gelatt, and M. P. Vecchi. 1983. Optimization by Simulated Annealing. Science 220, 4598 (1983), 671-680.

[10] M. Koibuchi and H. Matsutani and H. Amano and D. F. Hsu and H. Casanova. 2012. A case for random shortcut topologies for HPC interconnects. In 2012 39th Annual International Symposium on Computer Architecture (ISCA). 177-188. https://doi.org/10.1109/ISCA.2012.6237016

[11] Nicholas Metropolis, Arianna W. Rosenbluth, Marshall N. Rosenbluth, Augusta H. Teller, and Edward Teller. 1953. Equation of State Calculations by Fast Computing Machines. The fournal of Chemical Physics 21, 6 (1953), 1087-1092.

[12] Michihiro Koibuchi and Ikki Fujiwara and Kiyo Ishii and Shu Namiki and Fabien Chaix and Hiroki Matsutani and Hideharu Amano and Tomohiro Kudoh. 2016. Optical network technologies for HPC: computer-architects point of view. IEICE Electronics Express 13, 6 (2016), 20152007-20152007. https://doi.org/10.1587/elex.13.20152007

[13] Michihiro Koibuchi and Ikki Fujiwara and Shoichi Hirasawa and Satoshi Fujita and Koji Nakano and Takeaki Uno and Takeru Inoue and Ken-ichi Kawarabayashi. 2018. Graph Golf: The Order/degree Problem Competition. (2018). http://research.nii.ac.jp/graphgolf.

[14] NetworkX developers. $2018 . \quad$ NetworkX.

(2018). https://networkx.github.io.

[15] Nobutaka Shimizu and Ryuhei Mori. 2016. Average Shortest Path Length of Graphs of Diameter 3. CoRR abs/1606.05119 (2016). arXiv:1606.05119 http://arxiv.org/abs/1606.05119

[16] R. Mizuno and Y. Ishida. 2016. Constructing large-scale low-latency network from small optimal networks. In 2016 Tenth IEEE/ACM International Symposium on Networks-on-Chip (NOCS). 1-5. https://doi.org/10.1109/NOCS.2016.7579336

[17] R. Seidel. 1995. On the All-Pairs-Shortest-Path Problem in Unweighted Undirected Graphs. Journal of Computer and System Sciences 51, 3 (1995), 400-403. https://doi.org/10.1006/jcss.1995.1078

[18] Shin, Ji-Yong and Wong, Bernard and Sirer, Emin Gün. 2011. Smallworld Datacenters. In Proceedings of the 2Nd ACM Symposium on Cloud Computing (SOCC '11). ACM, New York, NY, USA, Article 2, 13 pages. https://doi.org/10.1145/2038916.2038918

[19] Teruaki Kitasuka and Masahiro Iida. 2016. A Heuristic Method of Generating Diameter 3 Graphs for Order/Degree Problem. CoRR abs/1609.03136 (2016). arXiv:1609.03136 http://arxiv.org/abs/1609.03136

[20] Warshall, Stephen. 1962. A Theorem on Boolean Matrices. F. ACM 9, 1 (Jan 1962), 11-12. https://doi.org/10.1145/321105.321107 